\title{
Electrical and optical characterization of Os-substituted rare-earth orthoferrite YbFe03-gamma powders
}

POLAT, Ö.; COSKUN, M.; SOBOLA, D.; KURT, B.; CAGLAR, M.; TURUT, A.

Applied Physics A: Materials Science and Processing

2021, vol. 127, iss. 1, pp. 1-11

ISSN $1432-0630$

DOI: http://dx.doi.org/10.1007/s00339-020-04182-1

Accepted manuscript

This is a post-peer-review, pre-copyedit version of an article published in Applied Physics A: Materials Science and Processing. The final authenticated version is available online at: 


\title{
Electrical and Optical Characterization of Os substituted Rare earth orthoferrite $\mathrm{YbFeO}_{3-\gamma}$ Powders
}

\author{
O. Polat ${ }^{1,2^{*}}$, M. Coskun ${ }^{3}$, D. Sobola ${ }^{1,4}$, B. Zengin Kurt ${ }^{5}$, \\ M. Caglar ${ }^{6}$, A. Turut $^{3}$ \\ ${ }^{1}$ CEITEC BUT, Brno University of Technology, Purkyňova 123, 61200 Brno, Czech \\ Republic \\ ${ }^{2}$ Institute of Physical Engineering, Brno University of Technology, Technická 2, \\ 61669 Brno, Czech Republic \\ ${ }^{3}$ Department of Physics, Faculty of Electrical Engineering and Communication, Brno \\ ${ }^{4}$ Faculty of Engineering and Natural Sciences, Department of Engineering Physics, \\ Istanbul Medeniyet University, 34700 Uskudar, Istanbul, Turkey \\ ${ }^{5}$ Faculty of Pharmacy, Department of Pharmaceutical Chemistry, Bezmialem Vakif \\ University, \\ 34093 Fatih, Istanbul, Turkey \\ ${ }^{6}$ Faculty of Science, Department of Physics, Eskisehir Technical University, 26000 Eskisehir, \\ Turkey
}

\begin{abstract}
The electrical properties of Os doped $\mathrm{YbFeO}_{3}(\mathrm{YbFO})$ powders prepared by solid-state reaction have been studied by Novocontrol Dielectric/Impedance Spectrometer. SEM, XPS and Raman spectroscopy were utilized for understanding chemical and structured analysis of the synthesized compounds. SEM images have revealed the void nature of the pellets. Furthermore, XPS studies have exhibited that $\mathrm{Yb}$ has $3+$ valance state. It is also revealed that the oxygen vacancies concentration drops as the Os doping level raises by XPS analysis. The frequency dependency of loss-tan $(\delta)$ examination has demonstrated that the $5 \mathrm{~mol} \%$ Os substituted sample has the lowest loss-tan $(\delta)$ values at high frequency regions at $100{ }^{\circ} \mathrm{C}$. It has been also realized that the $5 \mathrm{~mol} \%$ Os doped compound exhibits the highest resistivity among the samples. Raman spectroscopy examination has unveiled that the samples have similar space group. In addition, the optical band gap of the synthesized powders was also extracted via utilizing the Kubelka-Munk technique. It was realized that the band gap of YbFO slightly increases as the Os dopant ratio advances.
\end{abstract}

Key words: Solid state reaction; Os substitution; electrical properties; Raman spectroscopy; Optical bandgap

*Corresponding Author: ozgur.polat@ ceitec.vutbr.cz; ozgurpolat7@gmail.com 


\section{Introduction}

Rare earth orthoferrite, $\mathrm{RFeO}_{3}(\mathrm{R}=$ rare earth component), materials display novel electrical, optical and magnetic features and as a result of that they find different application areas in energy storage, spintronics, optical sensors and optical communications [1,2]. Furthermore, it has been shown that some of rare earth orthoferrites can exhibit both magnetic and ferroelectricity properties together, which make these materials new candidates in the multiferroic applications [3-6]. In $\mathrm{RFeO}_{3}$ structure, the bigger $\mathrm{R}$ cation is situated at the corner of unit cell, and $\mathrm{Fe}$ cations are placed at the center of octahedron $\mathrm{FeO}_{6}$ [7]. These materials display an orthorhombic structure with a space group of $\mathrm{Pbnm}$. $\mathrm{YbFeO}_{3}(\mathrm{YbFO})$ belongs to rare earth orthoferrites. Even though YbFO has orthorhombic structure, it is thin film form can have hexagonal phase, too $[8,9]$. Even though the magnetic properties of YbFO thin films have been extensively inspected, the electrical features of the same material have not attracted the same attention from the scientific community [10-12]. There are several groups including us have examined the electrical properties of YbFO material in ceramic form [13-16]. In addition, we have documented how the dopant affects the optical properties of YbFO thin films [17, 18]. Our studies have shown that the electrical and optical properties of the parental compound significantly influenced by oxidation state of the dopant, which determines the ionic radius. Because the dopant initiates crystal distortions and charge imbalance in the parent sample.

The present study is following up our previous study in which we have detailed the conductivity, electrical modulus, and dielectric constants of the undoped and Os doped YbFO powders [15]. In this manuscript, our objectives are to explore $i$ ) loss-tan( $\delta$ ), ii) impedance, $i i i)$ resistivity versus frequency at various temperatures, $i v$ ) Raman spectra, and $v$ ) the optical band gap of the synthesized samples. The mentioned electrical properties have detailed because 1) the loss tangent (dielectric loss tangent), tan $\delta$, is a parameter of a dielectric material that quantifies its inherent dissipation of electromagnetic energy at different frequencies. It exhibits the loss of electrical energy in the material via applied frequencies. On the other hand, the impedance spectroscopy is utilized to differentiate between transport characteristics of grains and grain boundaries in the material. In addition, the impedance spectroscopy is exploited to seek the electrical response of different phases and the dynamic behavior of localized and mobile charges in the bulk and interfacial regions in the samples. Consequently, the performance of grains, grain boundaries, electrode effects, different crystallographic entities and magnetic clusters can be investigated. It should also be mentioned that the Raman spectroscopy and optical band gap of the Os doped YbFO powders are introduced to the 
scientific community by the present investigation the first time. YbFO and Os doped $\mathrm{YbFO}$ powders have been obtained by well-known solid-state reaction method. X-ray photoelectron spectroscopy (XPS), scanning electron microscopy (SEM), Raman spectroscopy method were utilized to seek the chemical and structural analyses of the fabricated powders. Moreover, the electrical features of the compounds were studied with employing Broadband Dielectric/Impedance Spectrometer (Novocontrol Concept 50). Furthermore, the optical band gap undoped and Os doped YbFO powders have been discussed in the present study.

\section{Experimental}

The powder preparation was discussed in our previous study [15]. The microstructure of fabricated powders was studied by ULTRAPLUS ZEISS scanning electron microscope (SEM). Furthermore, X-ray photoelectron spectroscopy (XPS) (SPECS) was utilized to seek elemental composition of powders. For the calibration purpose, the $\mathrm{C} 1 \mathrm{~s}$ peak at $284.6 \mathrm{eV}$ peak was selected. In order to measure the electrical properties of synthesized compounds, their pellets were prepared by applying 10 tons of pressure with $13 \mathrm{~mm}$ diameter and sintering during four hours at $1150{ }^{\circ} \mathrm{C}$ in the atmospheric ambient. The thickness of the pellets is of $0.91 \mathrm{~mm}, 1.03$ $\mathrm{mm}$ and $0.88 \mathrm{~mm}$ for the undoped, $1 \%$ Os and $5 \%$ Os doped samples, respectively. The electrical characterizations of the pellets were performed using platinum $(\mathrm{Pt})$ electrode $(5 \mathrm{~mm}$ diameter). Broadband Dielectric/Impedance Spectrometer (Novocontrol Concept 50) was exploited for the electrical properties' investigations. The operating temperature gradually was varied from $-100{ }^{\circ} \mathrm{C}$ up to $100{ }^{\circ} \mathrm{C}$ and the applied frequency was swept from $1 \mathrm{~Hz}$ up to $10 \mathrm{MHz}$. Furthermore, WITec confocal Raman imaging system was used for collecting Raman spectra at room temperature. Laser with $532 \mathrm{~nm}$ excitation wavelength and $5 \mathrm{~mW}$ power was used at integration time of $10 \mathrm{sec}$. The optical band gaps of the fabricated films were investigated by UV-VIS Optical Spectrometer Ocean Optics JAZ 3-channel.

\section{Result and discussion}

\section{$\underline{3.1 \text { SEM and XPS Analysis }}$}

In our previous study, we have shown that the samples are single-phase with orthorhombic structure. In addition, we have not observed the formation of other phases. Furthermore, it has been exhibited that the lattice parameters of parent compound $\mathrm{YbFeO}_{3}$ increase as the Os dopant ratio raises, which is underlining the incorporation of Os into synthesized samples. The more detail about the crystal structure of undoped and Os doped YbFO powders can be seen in [15]. 
Figure 1 represents the surface topography of $\mathrm{YbFO}$ and Os doped $\mathrm{YbFO}$ pellets. The existence of agglomeration can be seen in Fig. 1 for all studied samples. It should also be stated that the examined samples exhibit the voids structure on the surface, which is consistent with their cross-sectional SEM images [15]. Such void structures underline the scrutinized samples display porosity nature.
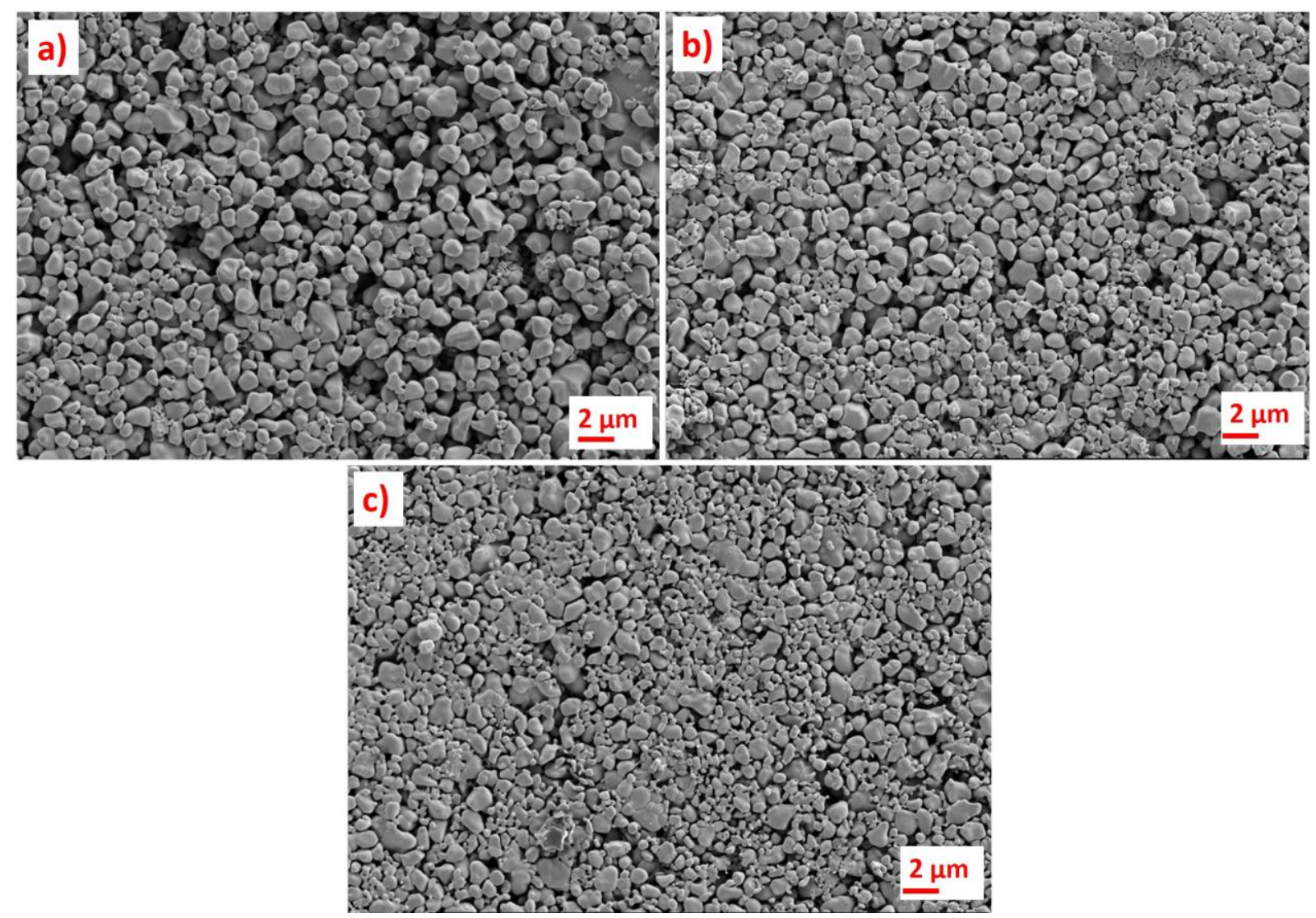

Fig. 1. SEM images of the examined samples: a) the undoped YbFO, b) $1 \%$ Os and c) $5 \%$ Os doped YbFO, respectively.

The oxidation levels of $\mathrm{Fe}$ and Os have been discussed in detail in our previous study [15]. Our XPS analysis, presented in [15], has revealed the valance states of Fe as 2+ and 3+. Moreover, the valance states of Os have been realized as 0 and 4+. It has been noted that the presence of $\mathrm{Fe} 2+$ has increased as the dopant level of Os is furthered. Such increase in the Fe 2+ might be connected to $i$ ) the oxygen vacancies, which are initiated because of high sintering temperature, ii) holding the charge balance owing to the presence Os atom in the structure [15 and references in it]. In this manuscript, the valence states of $\mathrm{Yb}$ and $\mathrm{O}$ elements are provided. Fig. 2 a) represents the doublet peaks of $\mathrm{Yb}$, which are appearing around $184 \mathrm{eV}$ and $200 \mathrm{eV}$. These doublet peaks exhibit that $\mathrm{Yb}$ has $3+$ oxidation state and those binding energies are close the literature values [19]. Figs. 2 b), c) and d) show the O 1s peak for the undoped, 1 mol and 5 mol 
$\%$ Os doped samples, respectively. From the figures, it is seen that $\mathrm{O} 1 \mathrm{~s}$ peak can be separated into two peaks, one is around $530 \mathrm{eV}$ that is associated to the lattice oxygen and other peak becomes visible about $531.3 \mathrm{eV}$, which is attributed to the oxygen deficiency/vacancy in the compound [20]. The Gaussian fitting unveils that the percentage of oxygen vacancies diminishes from $68.04 \%$ to $31.47 \%$ as the Os substitution is accelerated. In other words, the Os doped samples exhibit less oxygen vacancies than the undoped YbFO sample.
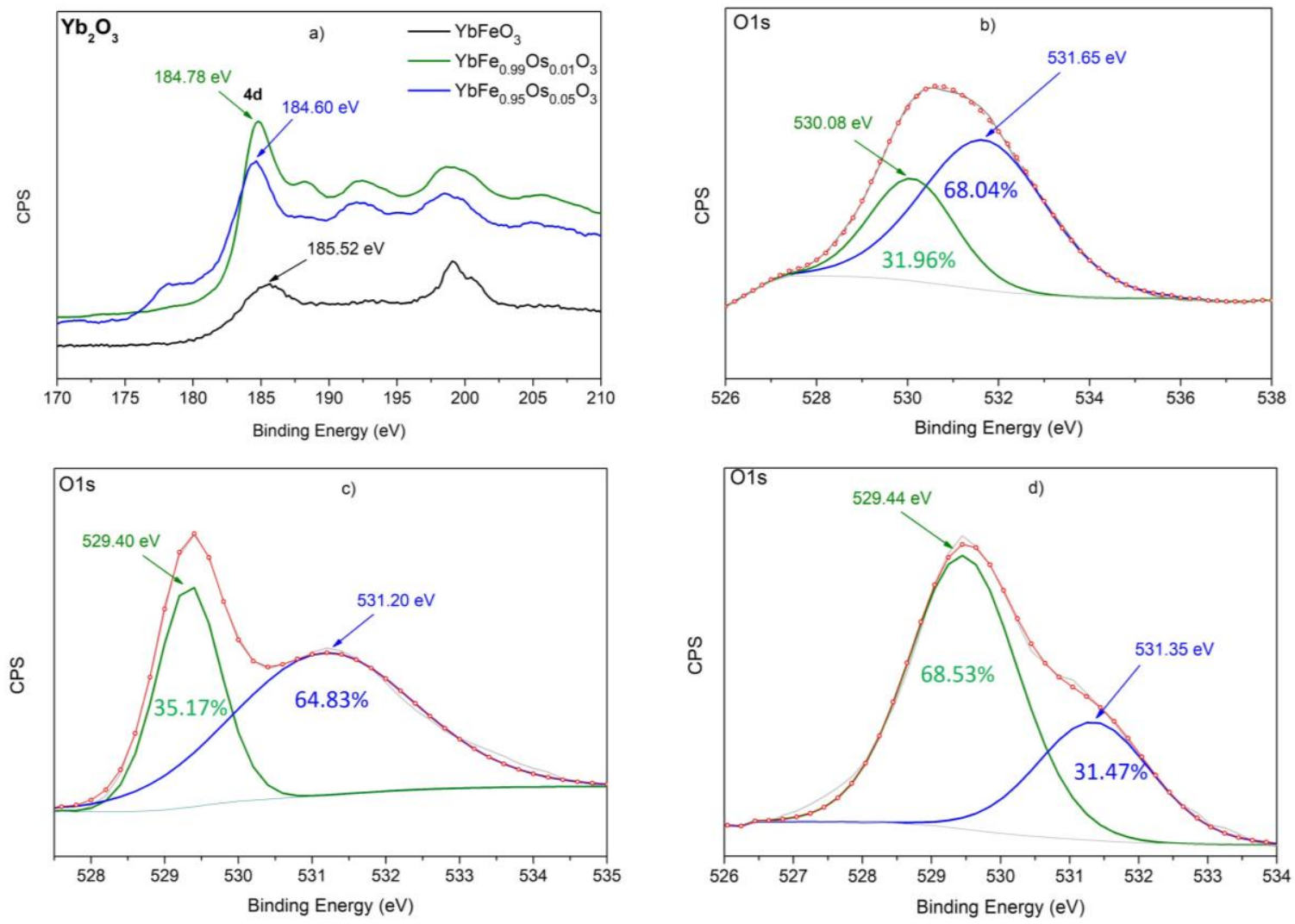

Fig. 2. XPS examination of YbFO and Os doped YbFO composites: a) Yb 4d spectrum for studied samples, $\mathrm{O}$ 1s spectrum of b) YbFO, c) $1 \mathrm{~mol} \%$ Os and d) $5 \mathrm{~mol} \%$ Os doped YbFO compounds.

Figure 3 represents the frequency dependence of loss-tan $(\delta), \tan (\delta)=\varepsilon^{\prime \prime} / \varepsilon^{\prime}$ here $\varepsilon^{\prime \prime}$ and $\varepsilon^{\prime}$ are the imaginary and real part of dielectric function, respectively, between $-100{ }^{\circ} \mathrm{C}$ and $100{ }^{\circ} \mathrm{C}$ with $20{ }^{0} \mathrm{C}$ increasing step. The loss-tangent is an important parameter of a dielectric material because it quantifies the dissipation of electromagnetic energy at different applied frequencies. Figure 3 a) displays the evolution of loss-tan $(\delta)$ values of the undoped YbFO with respect to applied frequency at various operating temperatures. It seems that the $\operatorname{loss}-\tan (\delta)$ has a maximum value (peak, which demonstrates the relaxation and represented by $\mathrm{f}_{1}$ ) around $10^{3} \mathrm{~Hz}$ at $-100{ }^{\circ} \mathrm{C}$ and $-80{ }^{\circ} \mathrm{C}$ and it lowers as the applied frequency is further boosted. On the other 
hand, when the temperature is in the range of $-60{ }^{0} \mathrm{C} \leq \mathrm{T} \leq 20{ }^{\circ} \mathrm{C}$, the initiation of two peaks become visible (they are denoted by $f_{1}$ and $f_{2}$ ). One peak appears at low frequency and other one shows up at higher frequency. Additionally, those peaks migrate to the advanced frequency as the temperature increases. Such behavior emphasizes the relaxation process is affected by temperature $[21,22]$. Yet, when the temperature is further advanced, $\mathrm{T} \geq 40{ }^{\circ} \mathrm{C}$, three relaxation peaks, one at (shown by $\mathrm{f}_{3}$ ) low and other two at high frequencies ( $f_{1}$ and $\mathrm{f}_{2}$, respectively), become eminent. It should be noted that the relaxation peaks shift to the higher frequencies as temperature goes up, Fig. 3 a). The progress of loss-tan $(\delta)$ values of $1 \mathrm{~mol} \%$ Os doped YbFO is denoted in Fig. 3 b) as function of frequency and temperature. The initiation of two small shoulders is seen at low and higher frequencies at $-100{ }^{0} \mathrm{C}$ and $-80{ }^{0} \mathrm{C}\left(\mathrm{f}_{1}\right.$ and $\mathrm{f}_{2}$ ). When the operating temperature is in the range of $-60{ }^{0} \mathrm{C} \leq \mathrm{T} \leq 0{ }^{0} \mathrm{C}$, one big relaxation peak (at low frequency region, demonstrated by $\mathrm{f}_{1}$ ) and small shoulder (at high frequency region, displayed by $\mathrm{f}_{2}$ ) can be observed. At $20{ }^{\circ} \mathrm{C}$ and $40{ }^{\circ} \mathrm{C}$ temperatures, only one visible relaxation peak is noted in the same figure. On the other hand, at higher temperatures, $60{ }^{\circ} \mathrm{C}, 80{ }^{\circ} \mathrm{C}$ and $100{ }^{\circ} \mathrm{C}$, one big peak (at high frequency, exhibited by $f_{2}$ ) and one small shoulder (at low frequency, denoted by $\mathrm{f}_{3}$ ) are noticeable, Fig. 3 b). It is also noted that the peaks are moving to the higher frequency values as the operating temperature is further accelerated. Nevertheless, in the case of $5 \mathrm{~mol} \%$ Os substituted sample, we have a different picture, Fig. $3 \mathrm{c}$ ). While one noticeable relaxation appears in the range of $-100{ }^{\circ} \mathrm{C} \leq \mathrm{T} \leq 60{ }^{\circ} \mathrm{C}$, no observation of relaxation peak at $\mathrm{T}$ $\geq 80{ }^{\circ} \mathrm{C}$ due to the instrument limitation. Figure $\left.3 \mathrm{~d}\right)$ compares the loss-tan $(\delta)$ values of the studied samples at $100{ }^{\circ} \mathrm{C}$. It is seen that at high frequencies $5 \mathrm{~mol} \%$ Os doped sample has lower $\operatorname{loss}-\tan (\delta)$ values. Such low loss-tan$(\delta)$ value makes the $5 \mathrm{~mol} \%$ Os doped sample a potential material for the future electronic device applications. Because of this potential, the real part of the permittivity of the $5 \mathrm{~mol} \%$ Os doped sample at various frequency and temperature are given in Table. 1.

Table 1. The real part of the permittivity (or dielectric constant) of the $5 \mathrm{~mol} \%$ Os doped sample at different frequency and temperature values.

\begin{tabular}{cccccccccccc}
\hline & $-100{ }^{\circ} \mathrm{C}$ & $-80{ }^{\circ} \mathrm{C}$ & $-60{ }^{\circ} \mathrm{C}$ & $-40{ }^{\circ} \mathrm{C}$ & $-20{ }^{\circ} \mathrm{C}$ & $0{ }^{\circ} \mathrm{C}$ & $20{ }^{\circ} \mathrm{C}$ & $40{ }^{\circ} \mathrm{C}$ & $60{ }^{\circ} \mathrm{C}$ & $80{ }^{\circ} \mathrm{C}$ & $100{ }^{\circ} \mathrm{C}$ \\
\hline $10 \mathrm{MHz}$ & 27,44 & 27,60 & 27,81 & 27,98 & 28,25 & 28,78 & 29,76 & 31,46 & 34,02 & 37,17 & 40,38 \\
$1 \mathrm{MHz}$ & 27,21 & 27,37 & 27,73 & 28,36 & 29,61 & 31,96 & 35,64 & 39,69 & 43,15 & 45,84 & 47,85 \\
$100 \mathrm{kHz}$ & 27,18 & 27,58 & 28,67 & 30,79 & 34,91 & 39,79 & 43,56 & 46,27 & 48,17 & 49,49 & 50,54 \\
$10 \mathrm{kHz}$ & 27,66 & 28,86 & 32,03 & 37,72 & 42,58 & 45,68 & 47,80 & 49,21 & 50,28 & 51,30 & 52,27 \\
$1 \mathrm{kHz}$ & 28,94 & 32,10 & 38,73 & 43,45 & 46,37 & 48,35 & 49,60 & 50,67 & 51,71 & 52,76 & 54,21 \\
$100 \mathrm{~Hz}$ & 32,42 & 38,75 & 43,64 & 46,51 & 48,57 & 49,84 & 50,96 & 52,04 & 53,31 & 55,58 & 60,02 \\
\hline
\end{tabular}


As it is noted from Fig. 3, loss-tan $(\delta)$ has larger values at low frequencies, which might be initiated due to $i$ ) the impact of interfacial loose and $i$ ) the presence of grain boundaries that diminish the mobility/migration of electrons between ions. It should be also noticed that as temperature goes up the loss-tan $(\delta)$ values advance. Because such high loss can be connected to charge exchange between $\mathrm{Fe}^{2+}$ and $\mathrm{Fe}^{3+}$ ions via resistive grain boundary.
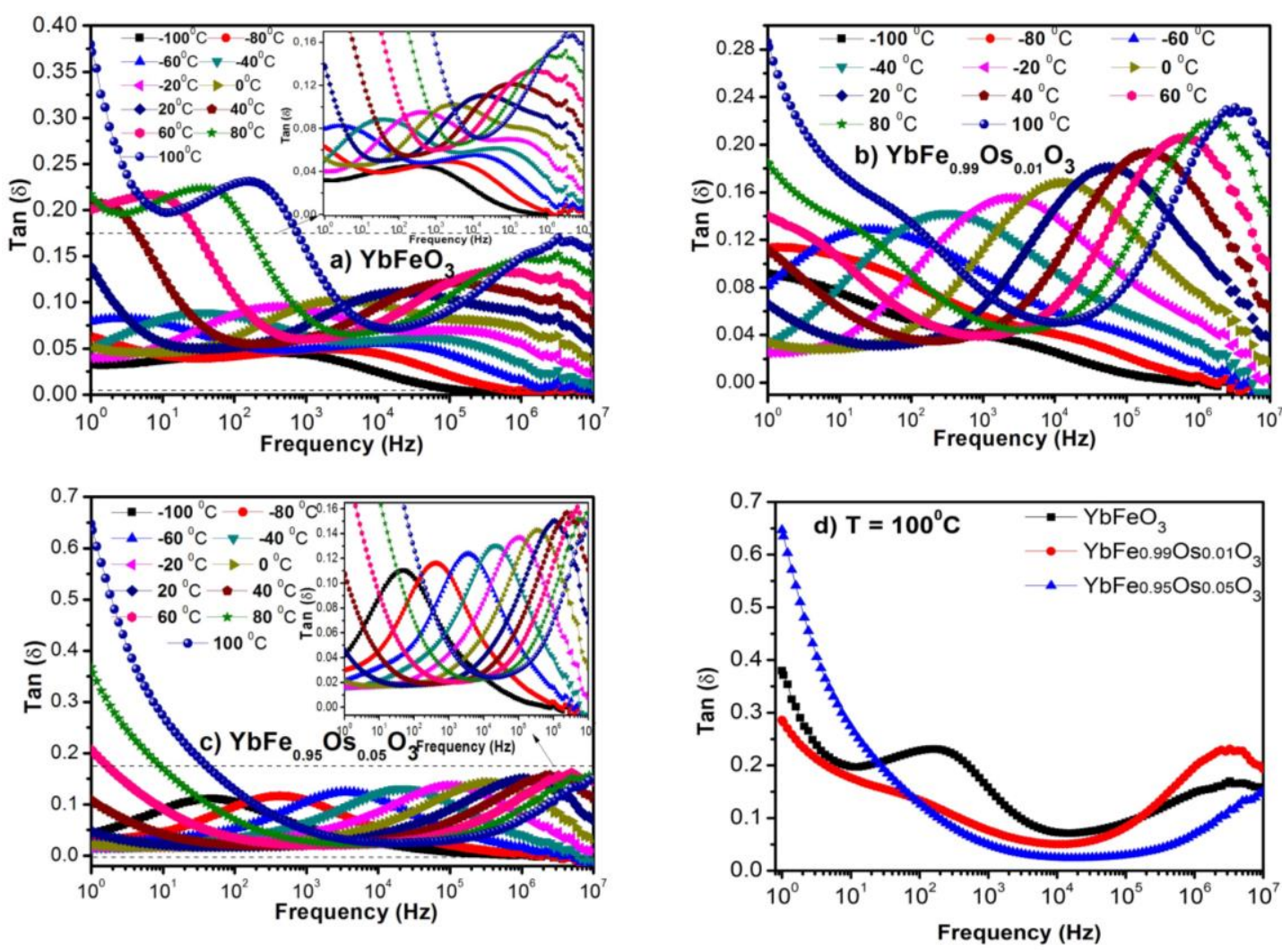

Fig. 3. The variation of $\tan (\delta)$ with respect to frequency and temperature for a) $\mathrm{YbFO}, \mathrm{b}) 1 \%$ Os and c) $5 \%$ Os doped YbFO specimens. d) represents the comparison of $\tan (\delta)$ at $100{ }^{\circ} \mathrm{C}$.

As it is mentioned above that the relaxation peaks move toward higher frequencies with operating temperature, which points out the temperature dependency of relaxation peaks. It is shown that such temperature dependency of relaxation peaks can be well described with Arrhenius:

$f_{\max }=f_{0} e^{\left(-\frac{E_{a}}{k_{B} T}\right)}$

here $f_{\max }, f_{0}, E_{a}, k_{B}$ and $T$ are the max. peak frequency of $\log f v s . \tan (\delta)$ plots, which can be extracted from Fig. 3, frequency dependent pre-exponential factor, activation energy, Boltzmann constant and the absolute temperature, respectively. The Arrhenius relation has been employed to calculate the activation energies of every individual relaxation peaks, which are 
presented by $\mathrm{f}_{1}, \mathrm{f}_{2}$ and $\mathrm{f}_{3}$. We have constructed Fig. $4\left(f_{\max } v\right.$ s. 1000/T) by utilizing $f_{\max }$ values from Fig. 3. It is seen that the undoped YbFO sample has three activation energy, depending on the relaxation type, Fig. 4 a). Here $f_{1}, f_{2}$ and $f_{3}$ represent the grain boundary, grain, and Maxwell-Wagner (effective at low frequency and high operating temperatures) relaxations, respectively. The obtained activation energies, $f_{0}$ and fitting $\mathrm{R}^{2}$ values of the undoped sample are shown in Fig. 4 a). It is seen that subtracted activation energies vary between $277 \mathrm{meV}$ and $849 \mathrm{meV}$. The calculated activation energies of $1 \mathrm{~mol}$ and $5 \mathrm{~mol} \%$ Os substituted samples are shown in Fig. 4 b) and c), respectively. The activation energies of Os doped samples vary between $233 \mathrm{meV}$ to $504 \mathrm{meV}$ depending upon the operating temperature. It can be concluded that the relaxation process taking place in the studied samples are due to oxygen vacancies whose presence has been shown in our XPS Figs. 2 b) - d). Because the activation energy of single and double ionized oxygen vacancies is 300 to $500 \mathrm{meV}$ and 600 to $1200 \mathrm{meV}$, respectively $[23,24]$.
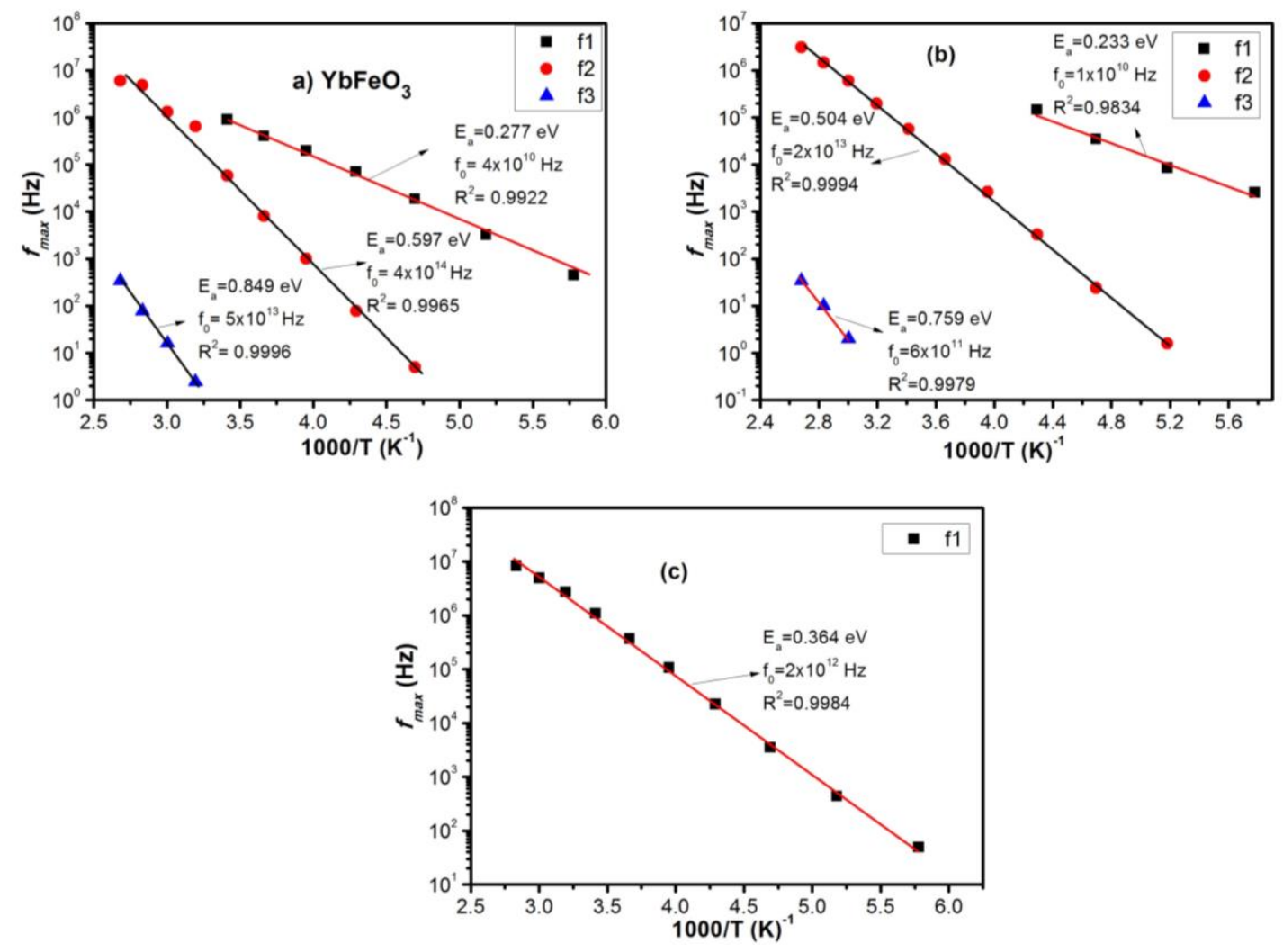

Fig. 4. $f_{\max } v s$. 1000/T plots for a) YbFO, b) $1 \mathrm{~mol} \%$ Os doped and c) $5 \mathrm{~mol} \%$ Os doped YbFO.

The impedance spectroscopy technique is employed to study the influence of grain boundary, grain, and electrode on the electrical features [25]. The complex impedance, $Z^{*}$, of a material is denoted by [26]; 
$Z^{*}=Z^{\prime}-j Z^{\prime \prime}=\frac{1}{j 2 \pi f \varepsilon^{*} C_{0}}=\frac{\varepsilon^{\prime \prime}-j \varepsilon^{\prime}}{2 \pi f C_{0}\left[\left(\varepsilon^{\prime}\right)^{2}+\left(\varepsilon^{\prime \prime}\right)^{2}\right]}$

here $Z^{\prime}, Z^{\prime \prime}, f, \varepsilon^{*}, C_{0}$ are the real and imaginary part of impedance, frequency, complex dielectric function and empty capacitance of the sample, respectively. The real part of impedance, $Z^{\prime}$, of the investigated samples versus frequency at different temperatures is given in Fig. 5 (semi-log scale). It is seen that the $Z^{\prime}$ values of the undoped and Os doped YbFO samples diminish as the applied frequency advances until couple of ten hertz at the all operating temperatures, then frequency independent area is initiated between $10^{2} \mathrm{~Hz}-10^{7} \mathrm{~Hz}$. As it is noted that when the applied frequency goes up the all curves start to overlap, and they form this frequency liberated region in which the energy barrier is reduced [27]. It should also be said that frequency free region extends as the operating temperature is escalated. Fig. $5 \mathrm{~d}$ ) compares the $Z^{\prime}$ values of the investigated compounds at $100{ }^{\circ} \mathrm{C}$. It is seen that the frequency independent region initiates around $20 \mathrm{~Hz}$ for the undoped and $1 \mathrm{~mol} \%$ Os doped samples, whereas the same region starts at slightly larger frequency, $\sim 35 \mathrm{~Hz}$, for the $5 \mathrm{~mol} \%$ Os substituted compound.
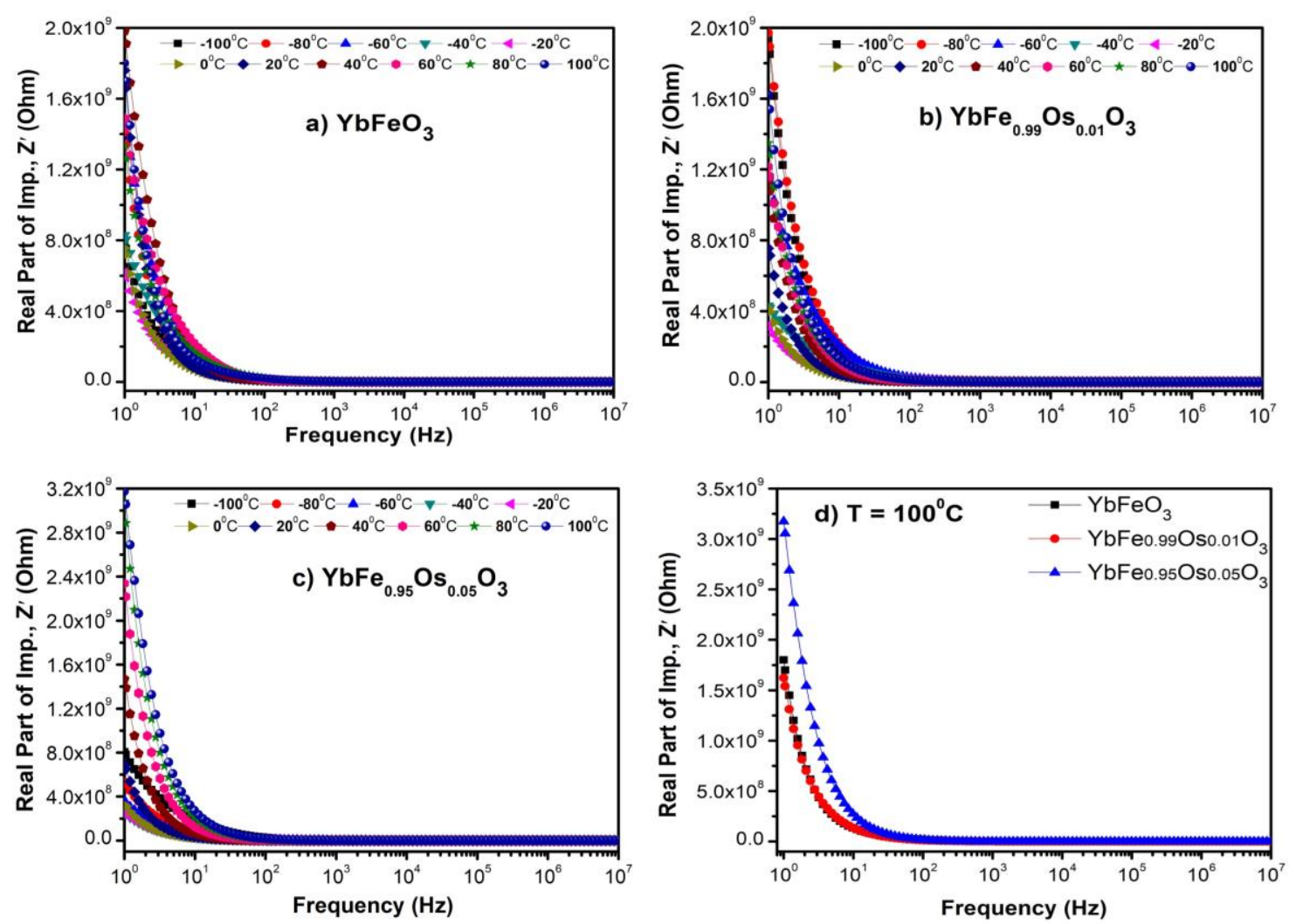

Fig. 5. Variation of real part of the impedance with respect to frequency and temperature for a) $\mathrm{YbFO}$, b) $1 \mathrm{~mol} \%$, c) $5 \mathrm{~mol} \%$ Os substituted YbFO. d) the real part of impedance of the studied samples is compared at $100{ }^{\circ} \mathrm{C}$. 
The frequency dependency of imaginary part of impedance, $Z^{\prime \prime}$, at various temperatures is given in Fig. 6 (semi log scale). It is seen that all studied samples exhibit similar behavior: at low frequency region $(0-20 \mathrm{~Hz}) \mathrm{Z}^{\prime \prime}$ decreases steeply and becomes frequency independent, forming frequency free region, as the frequency is advanced further. Such act might be ascribed to the buildup of space charges at higher frequency area [28]. Furthermore, as the operating temperature is enhanced this region prolongates to the lower frequency region. It should be stated that the formation of any relaxation peak has not been observed in the present compounds because of the instrument limitation.
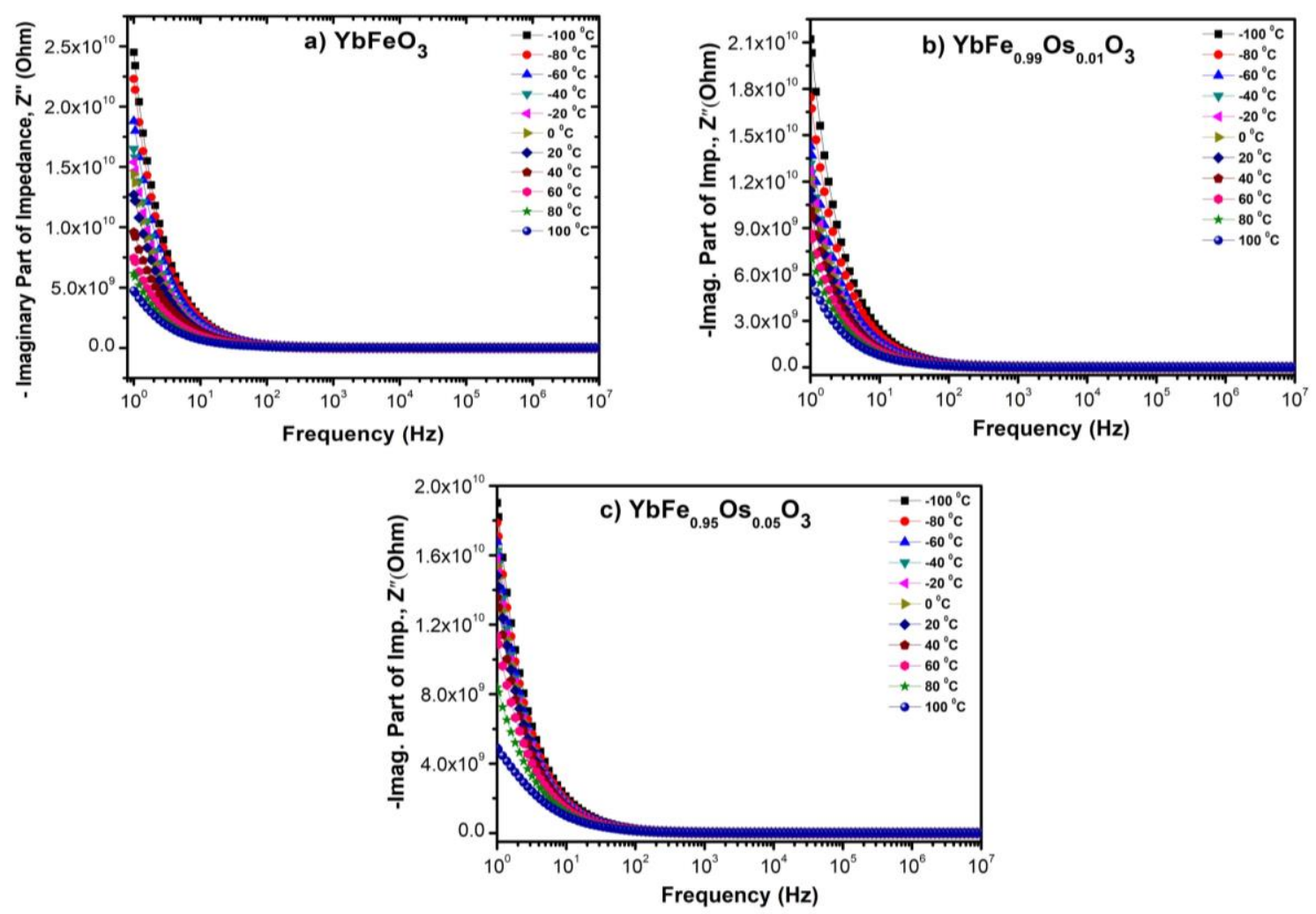

Fig. 6. The imaginary part of impedance variation at various frequencies and temperature values for a) YbFO, b) $1 \mathrm{~mol} \%$ Os and c) $5 \mathrm{~mol} \%$ Os substituted YbFO composites.

Figure 7 (semi-log scale) and inset figures (log-log scale) show the resistivity of the studied samples with respect to applied frequency at temperatures between $-100{ }^{\circ} \mathrm{C}$ until $100{ }^{\circ} \mathrm{C}$ with $20{ }^{\circ} \mathrm{C}$ steps. It is noted that the resistivity of samples diminishes with higher frequencies and temperatures. The same figures also represent that the investigated samples do not exhibit the formation of frequency free region, known as dc resistivity, at the operating frequencies and temperatures. In Fig. 7 d), the comparison of resistivity values of the examined samples can be seen at $100{ }^{\circ} \mathrm{C}$. It is seen that the $5 \mathrm{~mol} \%$ Os doped sample illustrates the highest resistivity 
(between $10^{2}$ and $10^{6} \mathrm{~Hz}$ ) among the studied samples. Such high resistivity of $5 \mathrm{~mol} \%$ Os doped sample could be related to $i$ ) less oxygen vacancy concentration. Our XPS examination has revealed that the concentration of oxygen vacancies decreases from $68.04 \%$ to $31.47 \%$ as Os doping ratio is boosted from 0 to $5 \mathrm{~mol} \%$ [29]. $\mathrm{ii}$ ) Os substitution can distort the crystal structure of $\mathrm{YbFO}$ owing to ionic radii difference between Fe and Os and such lattice distortion might lead to the formation of potential well trapping the charge carriers and increasing the resistivity [30].
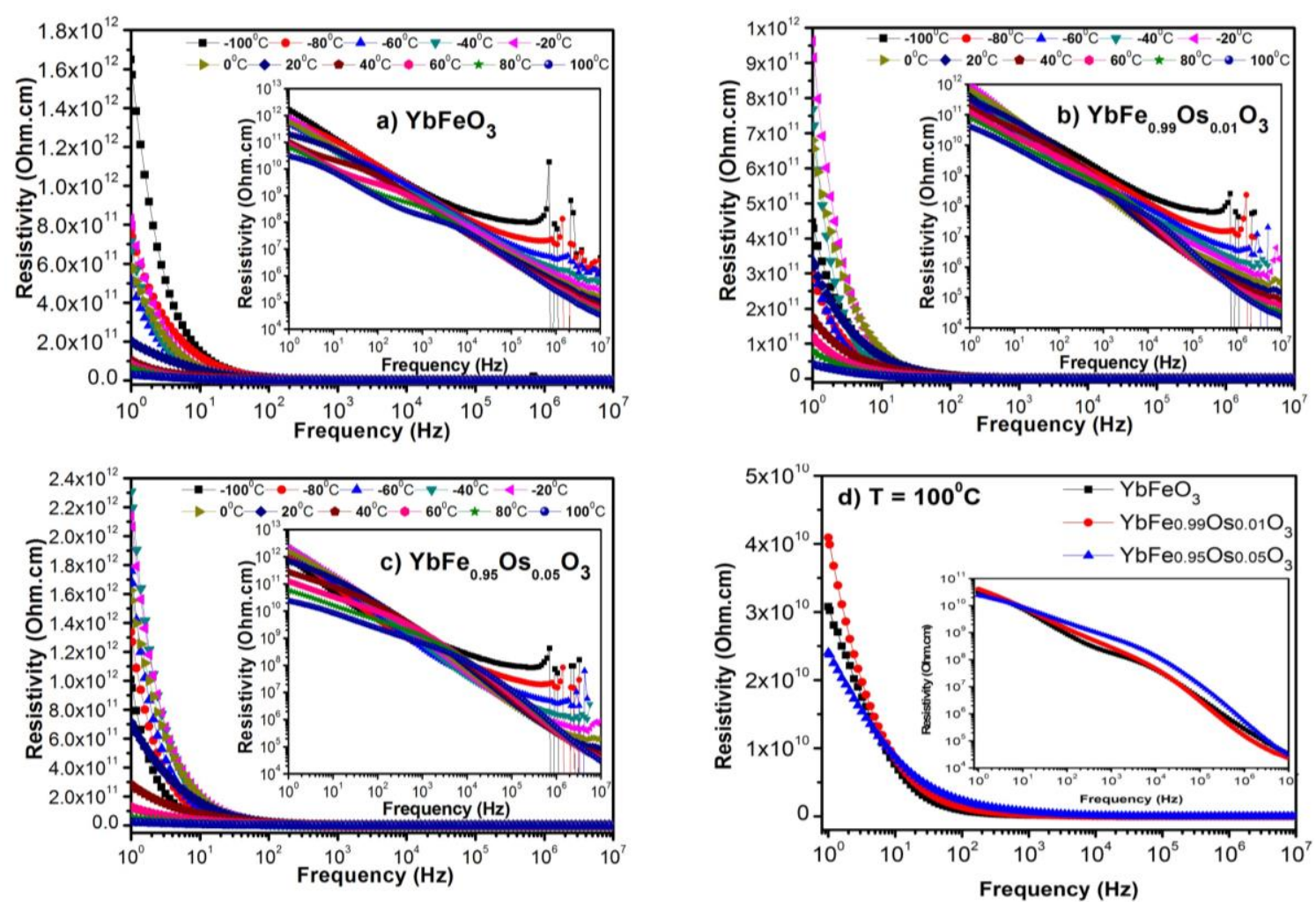

Fig. 7. Resistivity variation at different temperatures for a) YbFO, b) $1 \mathrm{~mol} \%$, c) $5 \mathrm{~mol} \%$ Os doped YbFO. d) The comparison resistivities of the investigated samples at $100{ }^{\circ} \mathrm{C}$.

The Raman spectra of the fabricated powders are provided in Fig. 8. In our previous study, we exhibited that $\mathrm{YbFeO}_{3}$ has orthorhombic Pnma structure [15]. Kroumova et al., have shown that orthorhombic Pnma structure has 24 Raman active vibrational modes [31], which are given by $\Gamma_{\mathrm{RA}}=7 \mathrm{~A}_{\mathrm{g}}+5 \mathrm{~B}_{1 \mathrm{~g}}+7 \mathrm{~B}_{2} \mathrm{~g}+5 \mathrm{~B}_{3 \mathrm{~g}}$ relation. The peaks below $200 \mathrm{~cm}^{-1}$ are assigned to the displacements of heavy rare-earth ions in the structure. The peaks appear at $269 \mathrm{~cm}^{-1}, 348 \mathrm{~cm}^{-}$ ${ }^{1}, 443 \mathrm{~cm}^{-1}$, and $509 \mathrm{~cm}^{-1}$ are associated to the excitation of magnetic $\mathrm{Fe}^{3+}$ ions [32]. The peak observed at $646 \mathrm{~cm}^{-1}$ might be linked to impurity scattering [33] or second order Raman scattering [34]. It is seen that the peak intensity increases as the Os substitution accelerates. 
Such increment in the intensity might be connected to $i$ ) the variation of grain size owing to Os dopant (Our previous XRD studies have shown that the grain size of YbFO increases from 43 $\mathrm{nm}$ to $53 \mathrm{~nm}$ with Os dopant [15]) and ii) oxygen vacancies. In addition to that a small shift to the higher wavenumbers can be noticed as the Os doping level is advanced. Such shift in the spectrum could be ascribed to the structural disorder initiated by Os substitution.

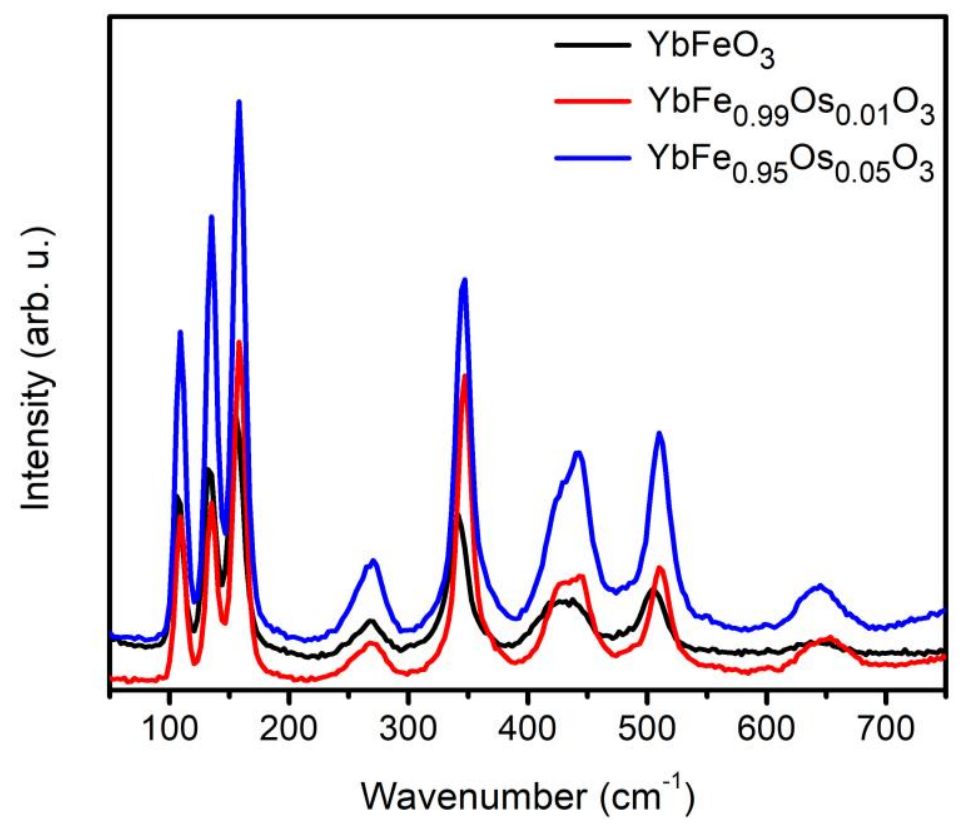

Fig. 8. Raman spectra for $\mathrm{YbFe}_{1-\mathrm{x}} \mathrm{Os}_{\mathrm{x}} \mathrm{O}_{3}(\mathrm{x}=0.00,0.01$ and 0.05$)$ samples.

The Kubelka-Munk technique has been employed to determine the optical band gap of powder samples or opaque films in the literature [35]. In this model, the data of diffuse reflectance $(R)$ spectrum is utilized. For this, $R$ spectra of all the obtained films were measured by taking a $\mathrm{BaSO}_{4}$ powder as a reference, at room temperatures. The optical band gap $\left(E_{g}\right)$ is identified via the following relation [35]:

$\left(\frac{F(R) h v}{t}\right)^{2}=A\left(h v-E_{g}\right)$

here $F(R), \mathrm{h} v, t$ and $A$ are Kubelka-Munk function $\left(F(R)=(1-R)^{2} / 2 R\right)$, the photon energy, the film thickness and a constant, respectively. The $E_{g}$ values of the obtained films estimated from the variation of the $(F(R) h v)^{2}$ with $h v$ are shown in Fig. 9. As it is noted, the $E_{g}$ values have been extracted via the extrapolation of the linear portion of the curves. It can be noticed that the optical band gap of YbFO slightly broadens, from $2.10 \mathrm{eV}$ to $2.2 \mathrm{eV}$, as the Os dopant level increases. The possible augmentation of the band gap might be associated to $i$ ) band gap 
tailing due to distribution of impurities/dislocations in the material. Particularly, band gap tailing is employed in polycrystalline samples to define the band spreading [36]. ii) MossBurstein effect $[37,38]$, occurring when the concentration of carriers dominates the conduction band edge density of states. Similar results have been obtained in the thin films of Os doped YbFO [39].

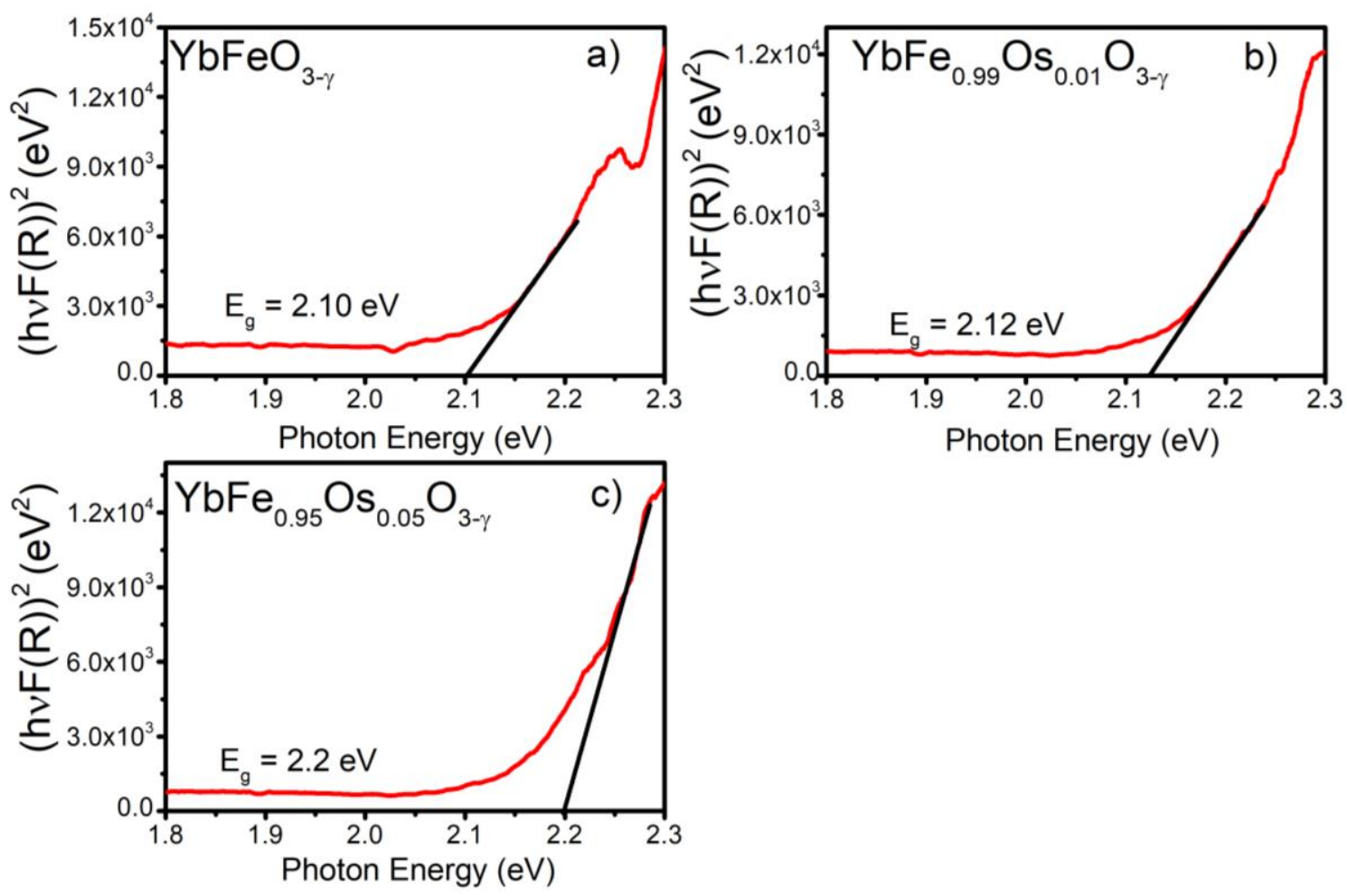

Fig. 9. Kubelka-Munk plots of the $\mathrm{YbFe}_{1-\mathrm{x}} \mathrm{Os}_{\mathrm{x}} \mathrm{O}_{3}(\mathrm{x}=0.00,0.01$ and 0.05$)$ samples.

\section{Conclusion}

The undoped and Os substituted YbFO powders were prepared via employing solid-state method. The pores structure of obtained powders was unleashed by SEM studies. XPS investigation exhibited that the concentration of oxygen vacancies reduces as the Os doping level advanced to $5 \mathrm{~mol} \%$. The $\operatorname{loss}-\tan (\delta)$ values of the studied samples were compared at 100 ${ }^{0} \mathrm{C}$ and it was demonstrated the $5 \mathrm{~mol} \%$ Os substituted compound holds the lowest loss-tan $(\delta)$ values. It was shown that at low frequencies the $\operatorname{loss}-\tan (\delta)$ has the highest values and such a behavior could be connected to $i$ ) the influence of interfacial loose and $i i$ ) the existence of grain boundaries that lessen the mobility/migration of electrons between ions. Furthermore, it was noted that the loss-tan $(\delta)$ values rise with the boosting operating temperature. The activation 
energies of investigated samples were subtracted from loss-tan $(\delta)$ with respect to applied frequency plots via utilizing Arrhenius relation. It turned out that oxygen vacancies dominate the relaxation process. In other words, the relaxation process occurs in the examined specimens are owing to oxygen vacancies because the extracted relaxation energies are close to the relaxation energies of oxygen vacancies in the literature. The impedance and resistivity studies underlined that $5 \mathrm{~mol} \%$ Os doped compound has higher resistivity among other samples. Raman investigation exhibited that the obtained compounds have similar space group. The present investigation has laid down that the electrical features of parent compound, YbFO, can be varied by replacing Fe sites with Os transition elements. It has been shown that the optical band gap of YbFO has increased from $2.1 \mathrm{eV}$ to $2.2 \mathrm{eV}$ as the Os substitution is elevated to 5 $\%$. Such widening in the band gap could be due to $i$ ) band gap tailing and ii) Moss-Burstein effect. The loss-tan $(\delta) v s$. frequency results at various temperature showed that the $\mathrm{YbFO}$ and Os doped YbFO compound could be a potential materials for the future electronic device applications due to the highly low dielectric loss.

\section{Acknowledgement}

This work was supported by The Scientific and Technological Research Council of Turkey (TUBITAK) through Grant No: 116F025. We acknowledge CEITEC Nano Research Infrastructure supported by MEYS CR (LM 2018110).

\section{$\underline{\text { References }}$}

[1] M. A. Ahmed, S.I. El-Dek, Optimizing the physical characterizations of orthoferrites to be used as pressure and gamma sensor. Materials Letters 60, 1437-1446 (2006)

[2] L. Li, X. Wang, Y. Lan, W. Gu, S. Zhang, Synthesis, Photocatalytic and Electrocatalytic Activities of Wormlike GdFeO3 Nanoparticles by a Glycol-Assisted Sol-Gel Process. Ind. Eng. Chem. Res. 52, 9130-9136 (2013)

[3] H. J. Zhao, Y. Yang, W. Ren, A.-J. Mao, X. M. Chen, L. Bellaiche, Creating multiferroics with large tunable electrical polarization from paraelectric rare-earth orthoferrites. J. Phys.: Condens. Matter 26, 472201 (2014)

[4] A. Stroppa, M. Marsman, G. Kresse, S. Picozy, The multiferroic phase of $\mathrm{DyFeO}_{3}$ : an ab initio study. New Journal of Physics 12, 093026 (2010) 
[5] K. F. Wang, J. M. Liu, Z. F. Ren, Multiferroicity: the coupling between magnetic and polarization orders. Adv. Phys. 58, 321 (2009)

[6] J.-H. Lee, Y. K. Jeong, J. H. Park, M.-A. Oak, H. M. Jang, J. Y. Son, J. F. Scott, SpinCanting-Induced Improper Ferroelectricity and Spontaneous Magnetization Reversal in $\mathrm{SmFeO}_{3}$. Phys. Rev. Lett. 107, 117201 (2011)

[7] Z. Zhou, L. Guo, H. Yang, Q. Liu, F. Ye, Hydrothermal synthesis and magnetic properties of multiferroic rare-earth orthoferrites. Journal of Alloys and Compounds 583, 21-31 (2014)

[8] A. A. Bossak, I. E. Graboy, O. Y. Gorbenko, A. R. Kaul, M. S. Kartavtseva, V. L. Svetchnikov, H.W. Zandbergen, XRD and HREM Studies of Epitaxially Stabilized Hexagonal Orthoferrites $\mathrm{RFeO}_{3}(\mathrm{R}=\mathrm{Eu}-\mathrm{Lu})$. Chem. Mater. 16, 1751-1755 (2004)

[9] M. S. V. Kumar, K. Kuribayashi, K. Kitazono, Effect of Oxygen Partial Pressure on the Formation of Metastable Phases from an Undercooled $\mathrm{YbFeO}_{3}$ Melt Using an Aerodynamic Levitator. J. Am. Ceram. Soc. 92, 903-910 (2009)

[10] H. Iida, T. Koizumi, Y. Uesu, K. Kohn, N. Ikeda, S. Mori, R. Haumont, P.E. Janolin, J.M. Kiat, M. Fukunaga, Y. Noda, Ferroelectricity and Ferrimagnetism of Hexagonal $\mathrm{YbFeO}_{3}$ Thin Films. J. Phys. Soc. Jpn. 81, 024719 (2012)

[11] H. Iida, T. Koizumi, Y. Uesu, Physical properties of new multiferroic hexagonal $\mathrm{YbFeO}_{3}$ thin film. Phase Transitions 84(9-10), 747-752 (2011)

[12] Y. K. Jeong, J.-H. Lee, S.-J. Ahn, S.-W. Song, H. M. Jang, H. Choi, J. F. Scott, Structurally Tailored Hexagonal Ferroelectricity and Multiferroism in Epitaxial $\mathrm{YbFeO}_{3}$ Thin-Film Heterostructures. J. Am. Chem. Soc. 134, 1450-1453 (2012)

[13] O. Polat, M. Coskun, F.M. Coskun, B. Zengin Kurt, Z. Durmus, Y. Caglar, M. Caglar, A. Turut, Electrical characterization of Ir doped rare-earth orthoferrite $\mathrm{YbFeO}_{3}$. Journal of Alloys and Compounds 787, 1212-1224 (2019)

[14] M. Coskun, O. Polat, F. M. Coskun, B. Zengin Kurt, Z. Durmus, M. Caglar, A. Turut, The Impact of Ir Doping on the Electrical Properties of $\mathrm{YbFe}_{1-\mathrm{x}} \mathrm{Ir}_{\mathrm{x}} \mathrm{O}_{3}$ Perovskite-Oxide Compounds. J. Mater. Sci: Mater. Electron. 31, 1731 (2020)

[15] O. Polat, M. Coskun, R. Kalousek, J. Zlamal, B. Zengin Kurt, Y. Caglar, M. Caglar, A. Turut, Frequency and Temperature-Dependent Electric Modulus Spectroscopy of Os Doped $\mathrm{YbFeO}_{3-\delta}$ Structure. J. Phys.: Condens. Matter 32, 065701 (2020) 
[16] O. Polat, M. Coskun, F. M. Coskun, J. Zlamal, B. Z. Kurt, Z. Durmus, M. Caglar, A. Turut Co doped $\mathrm{YbFeO}_{3}$ : exploring the electrical properties via tuning the doping level. Ionics $\mathbf{2 5}$, 4013-4029 (2019)

[17] O. Polat, M. Caglar, F. M. Coskun, M. Coskun, Y. Caglar, A. Turut, An investigation of the optical properties of $\mathrm{YbFe}_{1-\mathrm{x}} \mathrm{Ir}_{\mathrm{x}} \mathrm{O}_{3-\delta}(\mathrm{x}=0,0.01$ and 0.10$)$ orthoferrite films. Vacuum 173, $109124(2020)$

[18] O. Polat, M. Caglar F. M. Coskun, M. Coskun, Y. Caglar, A. Turut, An Experimental Investigation: The Impact of Cobalt Doping on Optical Properties of $\mathrm{YbFeO}_{3-\delta}$ Thin Film. Materials Research Bulletin 119, 110567 (2019)

[19] S. Ch. Sarma, U. Subbarao, Y. Khulbe, R. Jana, S. C. Peter, Are We Underrating RareEarth as an Electrocatalyst? The Effect of their Substitution in Palladium Nanoparticles Enhances the Activity towards Ethanol Oxidation Reaction. J. Mater. Chem. A. 5, 23369-23381 (2017)

[20] N. Paunovic, Z. D. Mitrovic, R. Scurtu, S. Askrabic, M. Prekajski, B. Matovic, Z. V. Popovic, Suppression of inherent ferromagnetism in Pr-doped $\mathrm{CeO}_{2}$ nanocrystals. Nanoscale 4, $5469-5476(2012)$

[21] S. Pattanayak, B.N. Parida, P. R. Das, R.N.P. Choudhary, Impedance spectroscopy of Gddoped $\mathrm{BiFeO}_{3}$ multiferroics. Appl. Phys. A 112, 387 (2013)

[22] A. K. Jonscher, The 'universal' dielectric response. Nature 267, 673 (1977)

[23] A. P. Barranco, J.D.S. Guerra, R. L.-Noda, E. B. Araujo, Ionized oxygen vacancy-related electrical conductivity in $\left(\mathrm{Pb}_{1-\mathrm{x}} \mathrm{La}_{\mathrm{x}}\right)\left(\mathrm{Zr}_{0.90} \mathrm{Ti}_{0.10}\right)_{1-\mathrm{x} / 4} \mathrm{O}_{3}$ ceramics. J. Phys. D: Appl. Phys. 41, $215503(2008)$

[24] J. Wu, J. Wang, Ferroelectric and impedance behavior of $\mathrm{La}-$ and $\mathrm{Ti}$ - co doped $\mathrm{BiFeO}_{3}$ thin films. J. Am. Ceram. Soc. 93, 2795-2803 (2010)

[25] V. Petrovsky, A. Manohar, F. Dogan, Dielectric constant of particles determined by impedance spectroscopy. J. Appl. Phys. 100, 014102 (2006)

[26] P.-F. Cheng, J. Song, Q.-P. Wang, S.-T. Li, J.-Y. Li, K.-N. Wu, Fine representation of dielectric properties by impedance spectroscopy. Journal of Alloys and Compounds 740, 36-41 (2018) 
[27] H. E. Sekrafi, A. B. J. Kharrata, M.A. Wederni, K. Khirouni, N. C.-Boudjadaa, W. Boujelben, Structural, electrical, dielectric properties and conduction mechanism of solgel prepared $\operatorname{Pr}_{0.75} \mathrm{Bi}_{0.05} \mathrm{Sr}_{0.1} \mathrm{Ba}_{0.1} \mathrm{Mn}_{0.98} \mathrm{Ti}_{0.02} \mathrm{O}_{3}$ compound. Materials Research Bulletin 111, 329337 (2019)

[28] A. R. James, K. Srinivas, Low temperature fabrication and impedance spectroscopy of PMN-PT ceramics. Mater. Res. Bull. 34, 1301 (1999)

[29] A. Kalabukhov, R. Gunnarsson, J. Börjesson, E. Olsson, T.Claeson, D. Winkler, Effect of oxygen vacancies in the $\mathrm{SrTiO}_{3}$ substrate on the electrical properties of the $\mathrm{LaAlO}_{3} / \mathrm{SrTiO}_{3}$ interface. Phys. Rev. B 75, 121404 (R) (2007)

[30] A. O. A. Keelani, S. Husain, W. Khan, Temperature dependent dielectric properties and ac conductivity of $\mathrm{GdFe}_{1-\mathrm{x}} \mathrm{Mn}_{\mathrm{x}} \mathrm{O}_{3}(0 \leq \mathrm{x} \leq 0.3)$ perovskites. J. Mater. Sci.: Mater. Electron. 30, 20119-20131 (2019)

[31] E. Kroumova, M. Aroyo, J. P.-Mato, A. Kirov, C. Capillas, S. Ivantchev, H. Wondratschek, Bilbao Crystallographic Server: Useful Databases and Tools for Phase-Transition Studies. Phase Transitions 76, 155-170 (2003)

[32] P. S. J. Bharadwaj, S. Kundu, V. S. Kollipara, K. B. R. Varma, Synergistic effect of trivalent $\left(\mathrm{Gd}^{3+}, \mathrm{Sm}^{3+}\right)$ and high-valent $\left(\mathrm{Ti}^{4+}\right)$ co-doping on antiferromagnetic $\mathrm{YFeO}_{3}$. RSC Adv. 10, 22183-22195 (2020)

[33] N. Koshizuka, S. Ushioda, Inelastic-light-scattering study of magnon softening in $\mathrm{ErFeO}_{3}$. Phys. Rev. B 22, 5394-5399 (1980)

[34] V. B. Podobedov, A. Weber, D. B. Romero, J. P. Rice, H. D. Drew, Effect of structural and magnetic transitions in $\mathrm{La}_{1-\mathrm{x}} \mathrm{M}_{\mathrm{x}} \mathrm{MnO}_{3}(\mathrm{M}=\mathrm{Sr}, \mathrm{Ca})$ single crystals in Raman scattering. Phys. Rev. B 58, 43-46 (1998)

[35] A. B. Murphy, Band-gap determination from diffuse reflectance measurement of semiconductor films, and application to photoelectrochemical water-splitting. Sol. Energ. Mat. Sol. Cells 91, 1326-1337 (2007)

[36] C. Rajashree, A. R. Balu, V. S. Nagarethinam, Properties of Cd doped PbS thin films: doping concentration effect. Surf. Eng. 31, 316-321 (2015)

[37] E. Burstein, Anomalous Optical Absorption Limit in InSb. Phys. Rev. 93, 632-633 (1954) 
[38] T. S. Moss, The Interpretation of the Properties of Indium Antimonide. Proc. Phys. Soc. Sect. B 67, 775-782 (1954)

[39] O. Polat, M. Caglar, F. M. Coskun, D. Sobola, M Konečný, M. Coskun, Y. Caglar, A. Turut, Examination of Optical Properties of $\mathrm{YbFeO}_{3}$ films via Doping Transition Element Osmium. Optical Materials 105, 109911 (2020) 